Supporting Information

\title{
Stern-Gerlach Study of Multidecker Lanthanide-Cyclooctatetraene Sandwich Clusters
}

\author{
Ken Miyajima, ${ }^{1}$ Mark B. Knickelbein, ${ }^{2}$ and Atsushi Nakajima*1,3
}

1 Department of Chemistry, Faculty of Science and Technology, Keio University,

3-14-1 Hiyoshi, Kohoku-ku, Yokohama 223-8522, Japan,

2 Chemistry Division and Center for Nanoscale Materials, Argonne National Laboratory, Argonne, Illinois 60439, USA

3 CREST, Japan Science and Technology Agency (JST), clo Department of Chemistry,

Keio University, Yokohama 223-8522, Japan

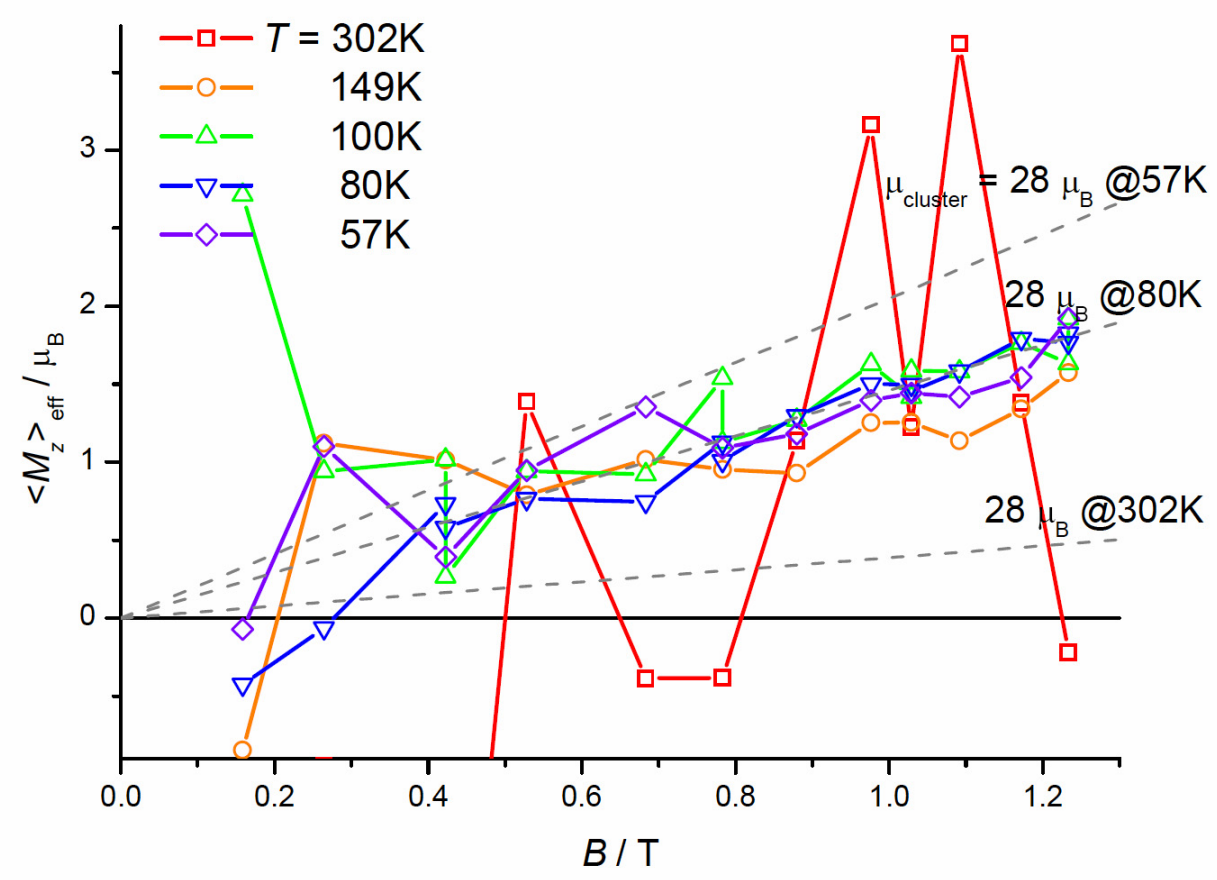

Supplemental Figure 1. Effective magnetic moment $\left\langle M_{\mathrm{Z}}\right\rangle_{\text {eff }}$ for $\mathrm{Ho}_{3}\left(\mathrm{C}_{8} \mathrm{H}_{8}\right)_{4}$ cluster derived from different temperatures. Dashed lines are the simulated slopes for corresponding temperatures. Although the experimental error is large for higher temperature, linear upward trend shows the validity for applying the superparamagnetic picture for $\mathrm{Ho}_{3}\left(\mathrm{C}_{8} \mathrm{H}_{8}\right)_{4}$. 

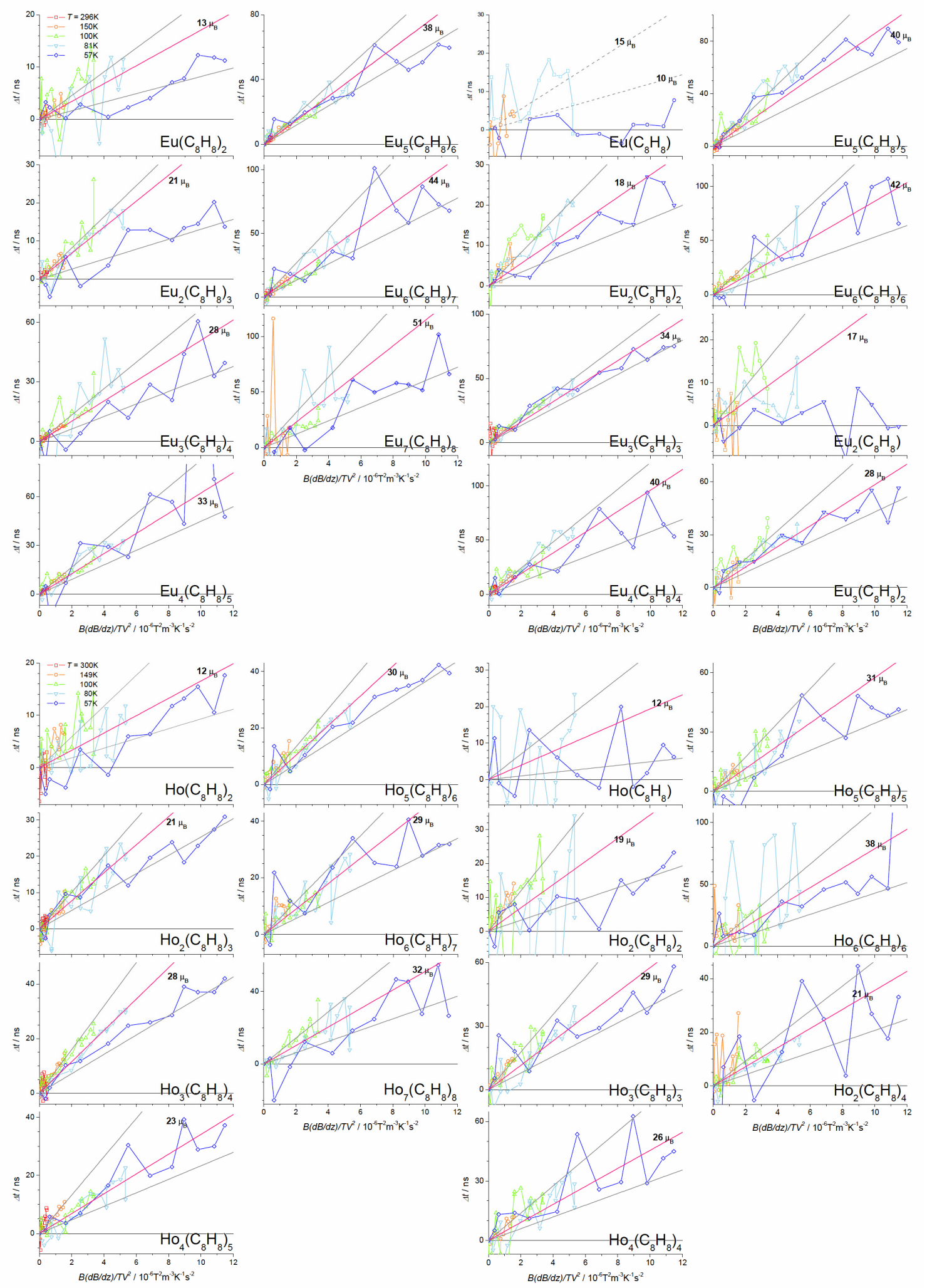

Supplemental Figure 2. Caption is given in the next page. 


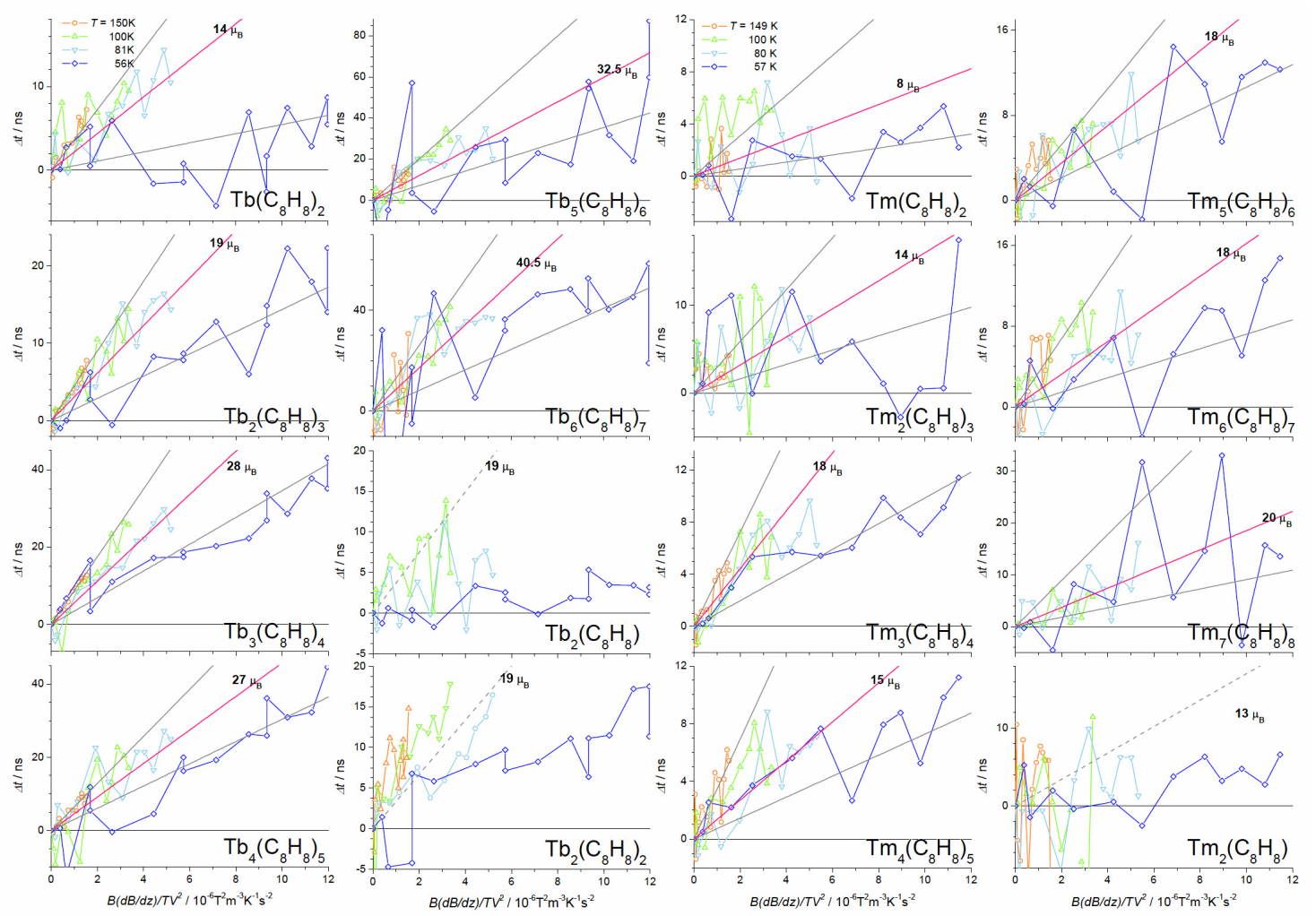

Supplemental Figure 2. Plots of magnetic deflection delay $\Delta t$ versus $B(\partial B / \partial z) /\left(T(V(T))^{2}\right)$ for Eu- $\mathrm{C}_{8} \mathrm{H}_{8}, \mathrm{~Tb}-\mathrm{C}_{8} \mathrm{H}_{8}, \mathrm{Ho}-\mathrm{C}_{8} \mathrm{H}_{8}$ and $\mathrm{Tm}-\mathrm{C}_{8} \mathrm{H}_{8}$ clusters. Each color corresponds to the cluster source temperatures. Most probable magnetic moment value at $T=80 \mathrm{~K}$ is depicted as solid lines. Gray colored solid lines indicate the upper and lower uncertainties of magnetic moments of the clusters, and gray colored dashed lines are displayed as references. 